\title{
SOFTWARE FOR SIMULATION OF BULK MEDIA FORCES ON AN EXCAVATION TOOL
}

\author{
Ahmad Hemami \\ Dept. Mining and Materials Eng. \\ McGill University, Montreal, Quebec \\ Canada H3A $2 A 7$ \\ $\underline{\text { Ahmad.hemami@mcgill.ca }}$
}

\begin{abstract}
A working code is developed to simulate the forces that are involved in the process of penetration of a bucket into bulk media during loading and excavation. The result of this work can be integrated with the simulation software that many manufacturers of heavy earthmoving machines introduce for training purposes. The simulator programs for machines such as backhoes, loaders, LHDs and the like, used for operator training, are usually only based on the kinematics of the machine. As a result, there is no indication of the effect of the medium when the cutting tool of such a machine touches the excavation material. This is like moving the machine in the air.

Feeling the resistance force of medium to cutting by a bucket or blade during loading and excavation is more realistic, both mentally (by vision) and physically (feeling resistance in pedals and levers). The results from force simulation can be used by feedback in creating simulated resistance in these training gears, similar to what is done in flight simulators for aircrafts.

In addition to serving for the above purpose, the results of this work can be used as a tool for examination and verification of the effect of a control policy for automation of loading and excavation of bulk media. So far no commercial product has been introduced that has such a capability, despite some extensive research on the subject.
\end{abstract}

\section{KEYWORDS}

Software, Simulation, Resistance force, Bulk media excavation, mining

\section{INTRODUCTION}

Nowadays, similar to a flight simulator for the training of pilots, for many earthmoving machines like a backhoe and grader, particularly the large ones, it is much more cost effective to train an operator on a simulator, first. A lot of progress has been made in this regard in terms of graphics and computer interaction. Nevertheless, these simulations are based on the kinematics of the machine, which translates into ignoring the dynamic effects. In particular, if the effect of interaction of the bucket (the excavating tool in general) with the earth is ignored the results is similar to moving the machine in the air.

In reality, when the excavating tool interfaces with the medium the force of interaction continuously changes and governs the motion and depth of penetration into the medium. If the training software is based on motion kinematics only, but lacks the behavior of the material during loading or excavation, the simulator gives a superficial feeling about the bucket action to the trainee, instead of a more natural feeling. For a more realistic simulation not only must this effect be embedded in the simulator code, it is also preferable if the pedals and levers of the rig be reflected with the effects of the interaction of the cutting tool and the medium.

In this work, a working code has been developed for simulation of the forces that are exerted from a medium to an excavator tool (backhoe and loader bucket, grader blade, etc). This code can be integrated with the software that is used in the training simulators for earthmoving machines. In 
addition, the code is usable for the simulation and verification of automation of earthmoving machines. A control policy can be evaluated or verified by simulation.

\section{EXCAVATION FORCE}

Hereafter the word tool will be used for the cutting element in an excavator, whether a blade, a bucket or other, depending on the machine. Also, the word excavator will be used for the earthmoving machine. When the tool of any excavator is in contact with a medium the cutting edge of the tool has to interface with the medium. So, the cutting edge encounters a force that must be overcome in order for the tool to penetrate into or cut through the medium. This force must be provided by the driving elements (actuators) of the excavator. This, however, is not the only force that must be overcome and must be provided by the actuators. Depending on the machine and the cutting tool form and size a number of resisting force components exist which have a resultant at the actuator level. The actuator, thus, must provide an active force superior to the total resistive force. The excavation force always comprises various components that vary in size and direction during excavation.

The excavation force depends on a number of parameters, (Hemami et al 1994). These parameters are determined by (1) operation [cutting, digging, scooping], (2) the tool [shape, sharpness, teeth], (3) medium (density, cohesion, average particle size, etc.) and (4) machine (grader, backhoe loader). Consequently, the simulation code is machine dependent.

For a bucket in a front-loader or a loader type excavator, for instance, these force components are as follows, shown in figure 1 (Hemami, 1994):

$\mathrm{r}_{1}$ : The weight of the material in the bucket (increasing as the bucket loads)

$\mathrm{r}_{2}$ : the force of the bucket body pushing (and compressing) the material

$r_{3}$ : Friction forces of the material moving into the bucket

$\mathrm{r}_{4}$ : Cutting force

In addition to the above, the active forces of the excavator must move the bucket, its contents and the material above the bucket that moves with the bucket. Moreover, the effects of the environment, more particularly the temperature and terrain slope, influence the force components. Proper adjustments, thus, becomes necessary.

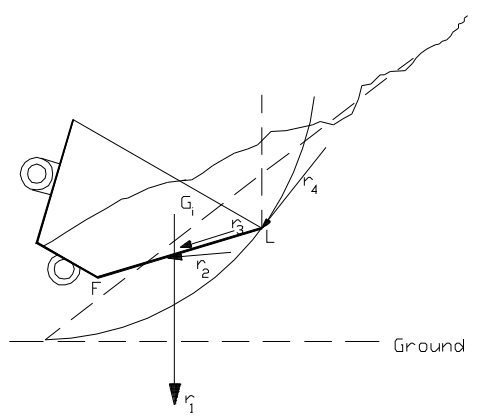

Figure1 - Forces from media on the bucket of a loader type excavator

As can be seen, the forces are considered in a planar motion for the bucket. This assumption greatly simplifies the relationships between the excavating force and the active forces from the exactor on its cutting tool provided by its actuators.

Analysis, formulation, and experimental measurement of excavation force components have been vastly studied by Zelenin et al (1985) in earlier decades and more recently a number of researchers have worked on the subject. See, for instance Blouin, et al (2001) and Takahashi et al (1999).

The research toward excavation automation and development of automated loading machines is the ultimate goal of numerous research works. The intention of this paper, however, is not to review the previous work, nor is it to list the many available references for various aspects of the required research. The references of the more recent aforementioned publications provide a sufficiently long list 
of contributors to the subject. For more references, see for instance, Richardson-Little, et al (2008), Vahed, et al (2008), and Ha, et al (2002).

\section{TOOL-MEDIA INTERACTION}

Since the excavation force depends also on tool motion, it is initially necessary to have the relationships between the tool motion and the machine actuators. The kinematic relationships for each type of machine can be determined by modeling as a robot manipulator based on the geometric and structure data. For example figure 2 illustrates the model for a loader and shovel bucket motion. These models, also for backhoe and some other machines, are available in the literature and are not discussed here.

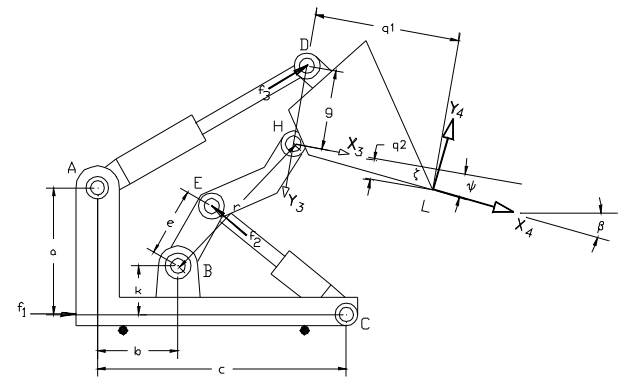

Figure 2- Robotic model of a loader

During the interaction of the active force from an excavator tool and the resistive force from the medium the behavior of the machine and the medium follow the physical laws. The machine has stiffness and damping that govern its deformation. The medium also undergoes a physical change. The medium can deform or displace with relatively little stiffness and very high damping.

A significant fact to realize and employ for simulation is that unlike two solid objects forcing each other in the interaction of a medium with a cutting tool, if the force from the tool is superior to the material resistance, motion takes place, otherwise there is no motion or it comes to a stop. In other words, the material cannot push the tool back if the tool force is inferior to the material resistance.

\section{MEDIA PROPERTIES}

The objective of this work is to focus on the resistance that a medium exhibits during excavation. This is the component $r_{4}$ in figure 1. For soil type material this is the dominant force among others, since it contains cutting and penetrating forces, unless a medium is light and can also easily pour into the bucket. Force $r_{4}$, moreover, depends on the properties of the excavated material. A number of properties have been listed in studying the bulk media and soil. Nevertheless, some are dependent on the others. Initially we can categorize bulk media into the following groups:

Homogenous or not homogenous (Soil can have large boulders in it)

Solid or particulate (Frozen soil is solid)

Cohesive or not cohesive (Cohesive material sticks to a tool; this can depend on the water content) Heavy or light (Soil is heavier than saw dust, for example). Compactness, porosity and shape of particles can all affect the density.

At this stage, in order to not get too involved with the many parameters, for the development of the code we have assumed that the material properties can be defined in the form of an array of nodes in two dimensions corresponding to the area to be excavated. Each node represents three values: a horizontal force, a vertical force and a torque. These are the three components of the resistance of the medium to excavation at that node. These values change from one node to the next and vary depending on the shape of the heap of material to be excavated. As well, the excavation force encountered by an excavating tool 
can significantly vary from point to point inside a medium. For this reason, at any node a random value is added to the nominal force/torque values.

In the simulations performed this randomly generated value is always positive, but its range of variation is quite large. Since the simulation is for the medium resistance, we are not concerned about the flexibility and damping of the structure of the bucket and its driving linkages at this stage.

In the computer code the number of nodes is increased in both dimensions, as desired, by interpolation. Thus, it is not necessary to have a large data input for a given medium and site. In the simulation, we assume the loading to take place from a heap of soil. Thus, the excavation force values depend on the location of the point of action inside the heap and, more importantly, if the bucket is driven out of the heap then the motion takes place in the air, for which there is no resistance. Figure 3 illustrates a typical heap, the desired tool trajectory and an example of the force nodes. The nodes in the air all have values of zero.

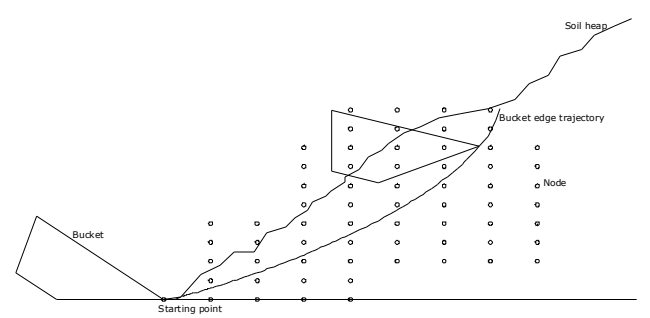

Figure 3- Schematic of a medium heap and force nodes representation

\section{PROCESS}

Figure 4 shows the major steps in developing the code. Some boxes are left blank on purpose because of the intellectual property right. In order to verify the code it is used to simulate the behavior of loading by a small loader from a small heap. The process of simulation consists of introducing a force (either fixed or variable) to each actuator and running into a heap from a medium whose properties are given in the form of force nodes, as discussed earlier. Then the movement of the bucket under the effect of the active forces to the excavator actuators is considered and recorded. In the process as the bucket moves and its orientation changes the corresponding force/torque elements on the cutting edge are continuously calculated and adjusted.

\section{SIMULATIONS}

The model loader used for simulation has two cylindrical actuators for raising and orienting the bucket plus a forward push. The bucket can be raised by $0.874 \mathrm{~m}$ from ground. A forward advance motion of $1.50 \mathrm{~m}$ is used for loading. There is $60^{\circ}$ orientation change from horizontal (start loading, orientation $\mathrm{b}=0$ ) configuration to the filled configuration $(b=60)$. The bucket motion is dictated by the lengths of the actuators at each instant. For the two cylindrical actuators if at any time the minimum or maximum length is reached no more retraction or extension can take place. The actuators' minimum and maximum lengths and their strokes, respectively, are:

Actuator 1: $\quad$ Min $=0.4049 \mathrm{~m}, \operatorname{Max}=0.7068 \mathrm{~m}$, stroke $=0.3019 \mathrm{~m}$

Actuator 2: $\quad$ Min $=0.7977 \mathrm{~m}, \operatorname{Max}=1.1017 \mathrm{~m}$, stroke $=0.304 \mathrm{~m}$

In the following examples the loader bucket is run into the medium without any control during the process, except that the force from each actuator is maintained or varies as planned. The outcome is, thus, a motion governed by the difference between the active forces provided by the actuators and the resistive forces at the bucket cutting edge transformed to the actuators. All the examples have the same starting configuration. Numbers shown in the figures that follow correspond to the bucket tip position in the vehicle's coordinate system. The starting point is lower than the vehicle frame. 


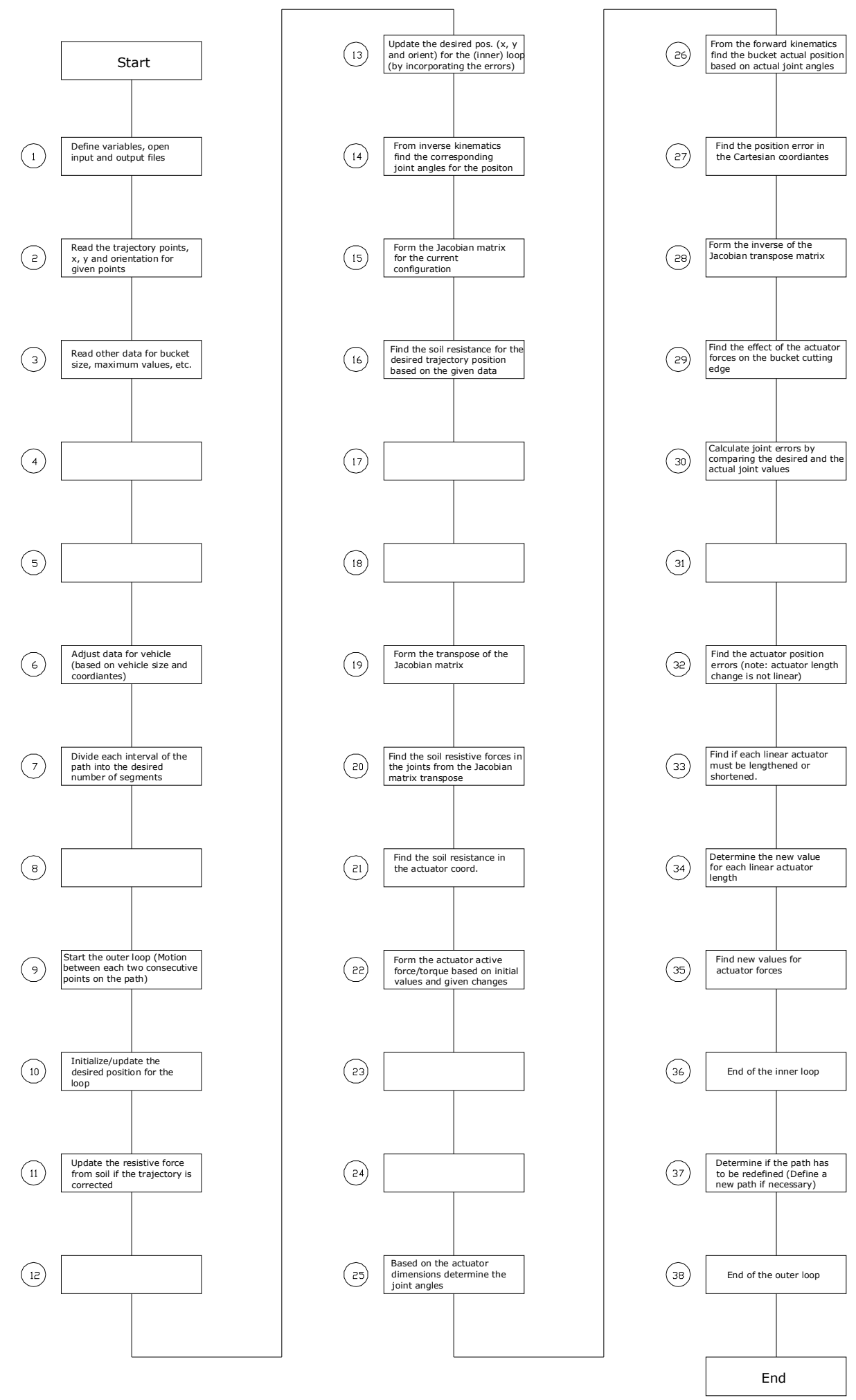

Figure 4- Steps in the simulation software 
For excavation automation all the active forces from the actuators must be controlled based on a control law, which yet is to be developed. The force inputs to the actuators, thus, are selected such that they demonstrate different scenarios. These forces can be smaller or larger, varying or constant and are not the same for the three actuators. Based on the expectation from what can physically happen as the forces act and the medium reacts it can be judged that these examples verify the validity of the code.

\section{Example 1-}

In this example the actuator forces (vehicle push, first cylinder push and second cylinder pull, respectively), are $2000 \mathrm{~N}, 1500 \mathrm{~N}$ and $1500 \mathrm{~N}$ from the beginning and stay constant for the whole time of simulation. When the bucket moves the effective mass values are increased by $10 \%$ for five times, evenly spaced during the period.

In this example the initial values of the actuator forces have not been sufficiently large to give rise to a noticeable motion of the bucket. The bucket has hardly changed orientation or moved in the vertical direction.

Figure 5 shows the position (x and y-coordinates) of the cutting edge of the bucket and its orientation at the starting point. The numbers represent the displacement in meters, in the horizontal and vertical directions. As is depicted by negative values shown in figure 5, the bucket has stayed below the vehicle platform, near the ground without being able to move up.

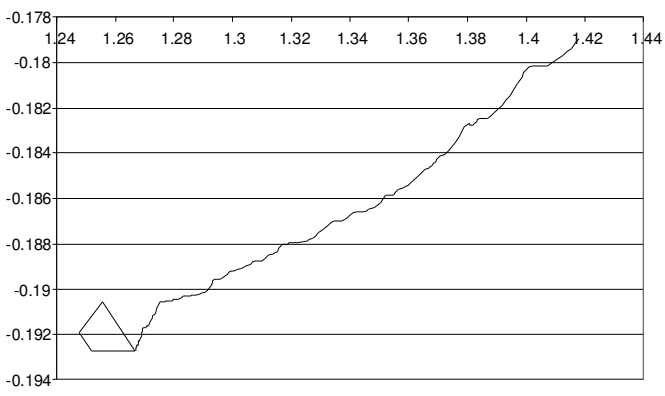

Figure 5 - Bucket motion in Example 1

\section{Example 2-}

For this example all the data is the same as before except for the active forces provided to the actuators that are increased five times by $20 \%$ in each step. The bucket movement and its orientation at a few points are shown in figure 6 . In this case the bucket has maneuvered a complete loading cycle by the time it is in the forth position shown. In this position, the linear actuators have reached their limits and, thus, the orientation has not changed afterwards, but the bucket has been pushed further forward. From the figure we may judge that during the first portion of the motion the forward push of the bucket has not been sufficient. Or in other words, the forces of the actuators 2 and 3 have been more than necessary.

\section{Example 3-}

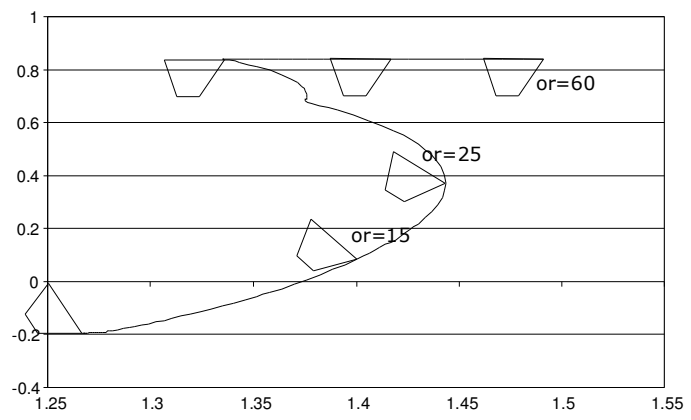

Figure 6 - Bucket motion for Example 2

In this example it is assumed that the same force scenario as in example 2 is used, with the exception that the material is heavier. The variable representing the material mass is five (5) times 
larger than those in example 2. Everything else (except the random values that determine the final resistive force) is the same as in example 2. The results are shown in figure 7 . The bucket has stopped at the final point shown; meaning that for the period the bucket could be moved only halfway.

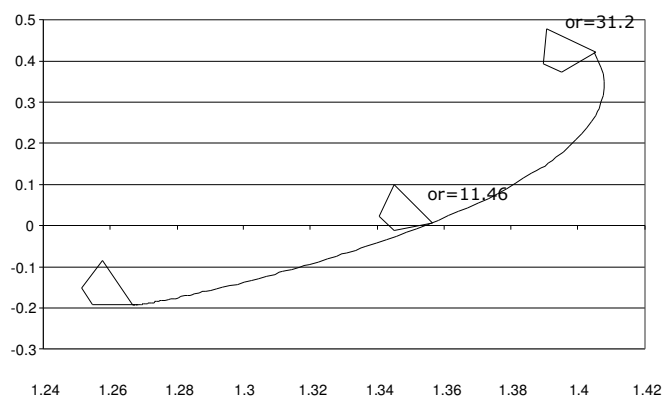

Figure 7 - Bucket motion for Example 3

\section{Example 4-}

Two changes have been introduced here compared to example 3. The stepwise increases in the actuators and mass have been modified from $20 \%$ and $10 \%$, respectively, to $33 \%$ and $20 \%$. The outcome is depicted in figure 8 .

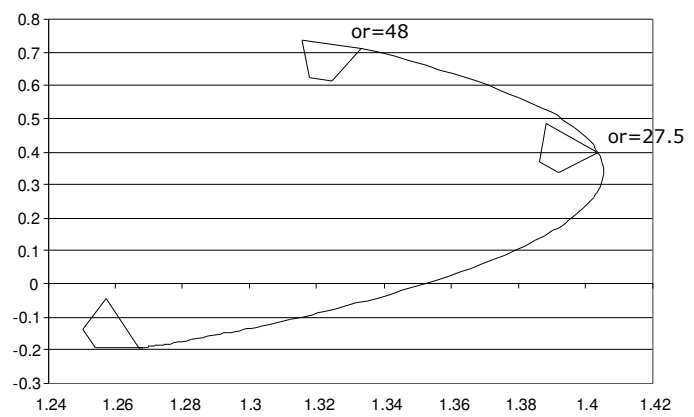

\section{Example 5-}

Figure 8 - Bucket motion for Example 4

The material density related values (mass representations as well as damping coefficients) have been further increased from their values in example 4; they have been doubled, for a heavier substance. The results are depicted in figure 9, which show a reasonable change (note that the motions for all the three degrees of freedom are smaller).

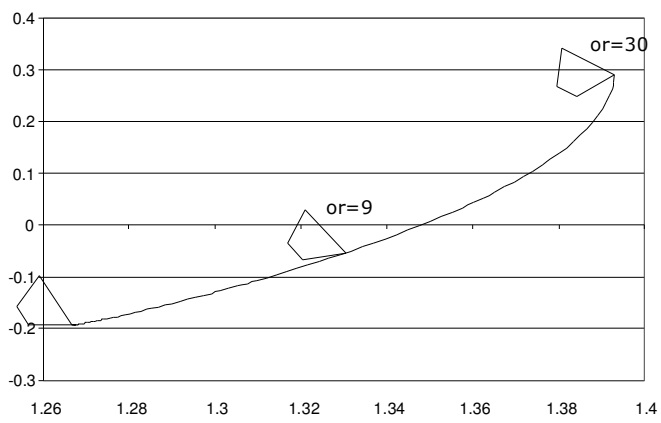

\section{Example 6-}

Figure 9 - Bucket motion for example 5

For the final example, we have kept all the characteristic data of example 5, except that the initial forces have all been increased by 200 units. As a result, the bucket has been moved further; particularly, the actions of the linear actuators are more pronounced. 


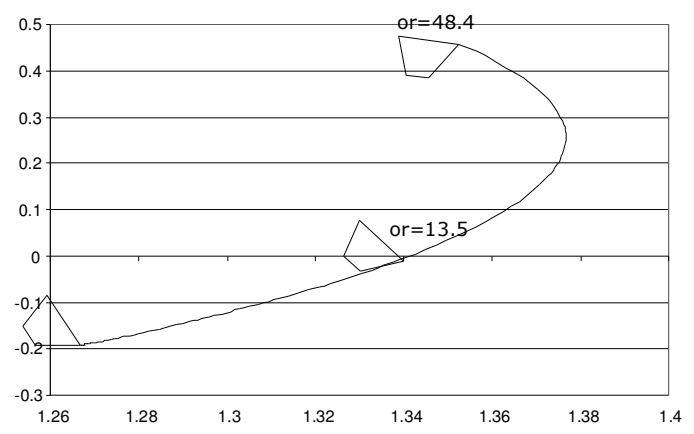

Figure 10 - Bucket motion for example 6

\section{SUMMARY}

This paper reports on a computer code that has been developed to simulate the interaction of media to excavation. Six examples are demonstrated each one of which represents a different scenario that can occur when a given set of forces is applied to the actuators of a small loader type excavator. The outcome of each scenario and a comparison of all the scenarios in terms of changes in the forces demonstrate reasonable results that one can expect from a physical device. The results, thus, demonstrate the validity of the developed computer code. This code can be successfully integrated with the training simulators of earthmoving machinery. Moreover, this code can be used for study of a control policy for the automation of an excavating machine.

\section{REFERENCES}

Blouin, S, Hemami, A and M. Lipsett, (2001) Review of Resistive Force Models for Earthmoving Processes, Journal of Aerospace Engineering, Vol. 14, No. 3, 102-111.

Ha, Q., Santos M., Nguyen, Q. Rye, D. and Durrant-Whyte, H., (2002), Robotic Excavation in Construction Automation, IEEE Robotics and Automation magazine, March 2002, 20-27

Hemami, A. Goulet, S. and Aubertin M., (1994), Resistance of Particulate Media to Excavation: Application to Bucket Loading, Int. Journal of Surface Mining and Reclamation, Vol 8, 125-129.

Hemami, A. (1994), Modelling, Analysis and Preliminary Studies for Automatic Scooping, Journal of Advanced Robotics, Vol. 8, No. 5, 511-529.

Richardson-Little W. and Damaren C. (2008), "Position Accommodation and Compliance Control for Robotic Excavation", Journal Aerospace Engineering, Vol. 21(1), 27-34

Takahashi, H., Hasegawa, M. and Nakano, E., (1999), Analysis of the resistive forces acting on the bucket of a Load-Haul-Dump machine and a wheel loader in the scooping task., Advanced Robotics, Vol.13 No. 2, 97-114.

Vahed, S.M., Song, S., Dai, J. S., Lam, H. K., Seneviratne L.D. and Althoefer K. (2008), “The Shortest Path of an Excavator Bucket in its Working Zone", Proc.17th IFAC World Congress (IFAC 08), Seoul (Korea), 13822-26

Zelenin, A. N., Balovnev, V. I. and Kerov, I. P., (1985), Machines for Moving the Earth, Amerind Publishing Co., New Delhi. 